\title{
Control for the Space Tether System Motion
}

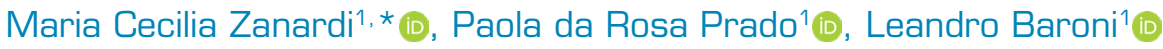 \\ 1.Universidade Federal do ABC - Centro de Engenharia, Modelagem e Ciências Sociais Aplicadas - Engenharia Aeroespacial - \\ Santo André/SP - Brazil. \\ *Correspondence author: mceciliazanardi@gmail.com
}

\section{ABSTRACT}

This paper proposed a study of a spatial tether system (STS), composed by two satellite (a main satellite and a subsatellite), with the objective of developing a control system in which the motion of the subsatellite is limited in the orbital plane of the main satellite. The linear quadratic regulator (LQR) method is used to implement this control, which is an optimal control with state feedback to predict the linearization of the equations of motion to calculate the feedback gain, using the resolution of Riccati equation. The results show an effective control, with the motion of the subsatellite limited only to the stretch of the cable that links both satellites. However, it is necessary to introduce an auxiliary torque, since the linearized equation associated with the second variation of the angle out of the plan does not have a term independent of the state vector.

Keywords: Spatial tether system; Control; LQR method.

\section{INTRODUCTION}

A space tether system (STS) is constituted of two or more rigid bodies in different orbits, connected by one or more flexible and compressed cables, commonly called tethers. These tethers are composed of a fibrous material highly resistant to normal traction, whose length can vary from a few hundred meters to tenths of kilometers, depending on the objective of the mission. To illustration in the Shuttle Electrodynamics Tether System (SETS) mission, in 1994, the length of the tether was $20 \mathrm{~km}$, and the YES2 mission (launched in 2007) deployed one of the longest tethers (31.7 km) (Cosmo and Lorenzini 1997). Space tether systems are not limited to satellites: an astronaut making external repairs at the International Space Station (ISS) is connected to it by a tether. This astronaut/tether/ISS system are considered an STS.

There are several applications for STS (Cosmo and Lorenzini 1997; Cartmell and McKenzie 2008; Chen et al. 2013), including electrodynamics propulsion, momentum exchange, artificial gravity, deployment of sensors or antennas, among others. To exemplify, some practical applications of these systems can be mentioned, emphasizing its importance. One of the main STS applications is found in the measurement of spatial geophysical gradients. A series of sub satellites $\left(\mathrm{S}_{2}, \mathrm{~S}_{3}, \mathrm{~S}_{4} \ldots\right)$ is distributed throughout a sole tether fixed to the space shuttle or at ISS, where the data collection would be made regarding different simultaneous altitudes. A very interesting application of STS is in aerodynamics. Using the space shuttle as a tug, it is possible to hold an experimental aircraft to a tether and study the aerodynamic effects over the real gas condition, without the issues found in common wind tunnels. One of the most important applications of STS is found in the electrodynamics field. The conducting and isolated tether movement within the geomagnetic field would be capable of generating a DC tension in the wire, which would feed the electric instrumentation on board the main satellite, at the cost of losing orbital energy. Plasma contactors would be fixed on both the tether edges to allow the generation of electric power. This last application of STS was put into practice in TSS missions that had

Received: Feb. 10, 2020 | Accepted: Oct. 10, 2020

Peer Review History: This is a peer reviewed version of selected paper presented at the $2^{\circ}$ Congresso Aeroespacial Brasileiro occurred in 16 -19 September of 2019 at Universidade Federal de Santa Maria, Santa Maria/RS, Brazil.

Section Editor: Marcelo Serrano Zanetti

This is an open access article distributed under the terms of the Creative Commons license. 
the goal of launching a satellite connected to a space shuttle through a tether, to make the data collection that help in the studies of space and plasmatic electrodynamics physics. The TSS-1 was the first space mission of the program, with its launching happening in July 1992, onboard the Atlantis Space Shuttle. The mission was successful and could create several information about the STS dynamic, showing that the tether can be released, controlled and recovered. Sufficient tensions were also generated through the tether and big ionosphere currents were extracted.

Other interesting applications are discussed in the following articles and involve the use of the STS as a slingshot and in maneuvers near asteroid. Lanoix and Misra (2012) discuss the application a tether slingshot combined with chemical propulsion for a near- Earth asteroid return mission. The combination of the chemical propulsion with slingshot leads to significant payload mass gains over conventional all chemical propulsion system. Ferreira et al. (2017a) performed a study of the dynamics of a system composed of a double asteroid where a tether is anchored in one of the bodies. The asteroids Ostro and Atiope (double and synchronous asteroids) are analyzed. Results show that the anchor point is in the plane formed by the bodies. A capture portal capable of altering the movement of a space vehicle is analyzed in Ferreira et al. (2017b) through a slingshot maneuver without spending fuel. This maneuver consists of a tether fixed on the surface of a body while the other end has a net, which will be performed by the vehicle, and can be used for capture or escape in relation to the main body. The use of this maneuver promotes a greater gain (or loss) of energy in relation to assisted gravitational maneuvers. Moia et al. (2019) presented the mathematical formulation for the system formed by two-point masses connected by a tether in the central force field, in a Keplerian movement. The Lagrangian formulation is used to describe the rotational motion of the dumbbell-like system. Results of tension and kinetic energy are presented for two different situations, considering equal masses and different masses.

In this paper, an STS composed of two satellites was considered (Zanardi et al. 2019), a main satellite $S_{1}$ with mass $m_{1}$ and a subsatellite $S_{2}$, with mass $m_{2}$, as illustrated in Fig. 1 . The main satellite $S_{1}$ orbits the Earth in a circular trajectory of $R$ radius. It is connected to a smaller subsatellite $\mathrm{S}_{2}$ by a rigid and inflexible tether, with length $l$ and mass $m_{\mathrm{t}}$. This tether can be expanded or retracted, making a $T$ tension arise in the wire. A similar STS was discussed by Denney and Greene (1991), Yong et al. (2009) and Zanardi et al. (2019).

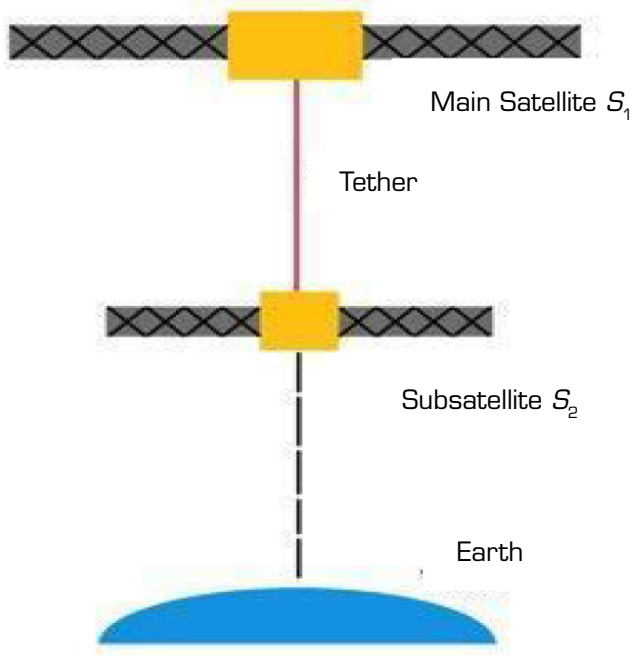

Figure 1. Configuration of an STS with two satellites (Denney and Greene 1991).

The equations that describe the translational and rotational motion of the subsatellite around the main satellite are presented in Denney and Greene (1991). They studied the effects of instrumentation accuracy and configuration on estimation error for the small expensive-tether deployment system using continuous-discrete extended Kalman filter state estimator. The results indicate that the originally planned instrumentation package could not estimate the state vector adequately. They recommend adding 
instruments and increasing the selected measurements accuracies to reduce the estimation error. The equations presented by Denney and Greene (1991), for the motion of the sub satellite are used in this paper.

To make the estimator more accurate, Yong et al. (2009) studied the estimation of tether angles and their rates. They simplified the translational equations of motion of the subsatellite and combined the measurements of a three-axis tether tensiometer with the information of the attitude sensor, to estimate these parameters in real time through the extended Kalman filter. The proposed estimator not only reduces the complexity of the system model, but also improves the parameter precision. Some data for the space tether system presented by Yong et al. (2009) are used in this paper.

The results of dynamic propagation associated with the motion of the subsatellite around the main satellite are shown in Zanardi et al. (2019). A $4^{\text {th }}$ Order Runge-Kutta method was used for the integration, with the MATLAB software. The results were compared to the results presented by Yong et al. (2009) in order to validate the data obtained by the propagator. The results have shown to be suitable, agreeing with the known data. It has been identified that the subsatellite has a counterclockwise precession movement in the orbital plane of the main satellite and that it oscillates vertically through this movement. The integration methods developed by Zanardi et al. (2019) are useful for the control process used in this paper.

The subsatellite motion around the main satellite requires the definition of two coordinate frames. The coordinate frame $F_{0}$, with origin in $O$ in the center of the mass $(\mathrm{CM})$ of the main satellite $\mathrm{S}_{1}$, and is related to its orbital movement, being that the axis $X_{0}$ points in the direction of the orbital speed vector and axis $Y_{0}$ points in the direction that connects the $S_{1}$ 's CM to the Earth's CM. Also, one coordinate frame $F$ with origin in the $\mathrm{CM}$ of the subsatellite $S_{2}$, whose axis directions are associated to the characteristics of $S_{2}$. System $F$ is nonrotating and coincides with the principal system of inertia of subsatellite $S_{2}$ in the initial instant.

To determine the subsatellite position regarding the main satellite, the spherical coordinates $(1, \alpha, \beta)$ are used: $\alpha$ represents the angle on the plane between the $O Y_{0}$ and the projection of the $S_{2}$ position in the plane $O Y_{0} X_{0}$, $\beta$ represents the angle out of the plane between $l$ and the projection of the $\mathrm{S}_{2}$ position in the plan $O Y_{0} X_{0}$, (Fig. 2). The tension in the tether $T$ is represented in the coordinates of system $F_{0}$.

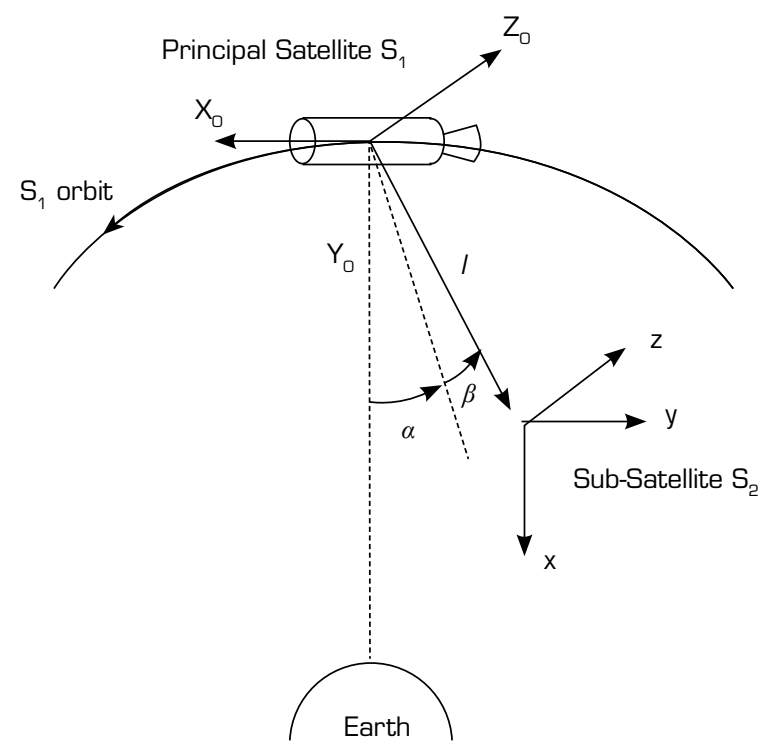

Figure 2. Characteristics of the analyzed STS (Denney and Greene 1991).

The goal of this paper is to present a study of a spatial tether system composed by the $S_{1}$ and $S_{2}$ satellites with the objective of developing a control system in which the motion of the $S_{2}$ is limited in the orbital plane $O X_{0} Y_{0}$ of the $S_{1}$.

The linear quadratic regulator (LQR) method is used to implement the control system for the subsatellite motion around the main satellite. This method consists of an optimal control technique with state feedback to predict the linearization of the equations 
of motion to calculate the feedback gain, using the resolution of Riccati equation. This method is applied to limit the motion of subsatellite in the main satellite orbital plane and along the tether.

\section{EQUATIONS OF MOTION}

According to Denney and Greene (1991), the equations of translational motion of the subsatellite $S_{2}$ around the main satellite $S_{1}$ are given by Eq. $1-3$ :

$$
\begin{gathered}
\frac{d^{2} l}{d t^{2}}=M_{21} l \\
\frac{d^{2} \alpha}{d t^{2}}=-2 \frac{M_{23}}{l} \frac{d l}{d t}\left(\frac{d \alpha}{d t}+\frac{d w}{d t}\right)-2 \frac{d \beta}{d t}\left(\frac{d \alpha}{d t}+\frac{d w}{d t}\right) \tan (\beta)-3 \\
\frac{d^{2} \beta}{d t^{2}}=-2 \frac{M_{23}}{l} \frac{d l}{d t}\left(\frac{d \beta}{d t}\right)-\left(\frac{d \alpha}{d t}+\frac{d w}{d t}\right)^{2} \operatorname{sen}(\beta) \cos (\beta)-3
\end{gathered}
$$

where: $w$ is the latitude argument defined by the sum of perigee argument and the true anomaly, associated with the orbital movement of the main satellite $S_{1}, \mu$ is the Earth's gravitational parameter, $T$ is the tensor in the tether, $R$ is the radius of the circular orbit of $S_{1}$ around the Earth assumed equal to $6878 \mathrm{~km}$ (Yong et al. 2009) and $M_{21}, M_{23}$ and $\frac{d w}{d t}$ are defined by Eq. 4 :

$$
M_{21}=\frac{m_{2}+\frac{m_{t}}{2}}{m_{2}+m_{t}}, \quad M_{23}=\frac{m_{2}+\frac{m_{t}}{2}}{m_{2}+\frac{m_{t}}{3}} \quad, \quad \frac{d w}{d t}=\sqrt{\frac{\mu}{R^{3}}}
$$

Yong et al. (2009) assumed a variation law to the length of tether $(l)$, which is not dependent of others state variables $\left(\alpha, \beta, \frac{d \alpha}{d t}, \frac{d \beta}{d t}\right)$, with the final length $l_{\text {final }}=45 \mathrm{~km}$, initial length $l_{\text {inicial }}=50 \mathrm{~km}$ and $c$ a constant equal to 0.005 :

$$
l=l_{\text {final }}+\left(l_{\text {inicial }}-l_{\text {final }}\right) e^{-c t}
$$

Assuming the variation law established by Eq. 5 for the tether length, the motion equations are summarized in Eqs. 2 and 3. Thus, Eqs. 2, 3 and 5 are used to describe the motion of the subsatellite around the main satellite, and Eqs. 2 and 3 must be linearized for the application of the LQR method.

\section{LINEAR REGULATOR QUADRATIC CONTROL (LQR)}

Consider a linear system described by Eq. 6:

$$
\frac{d X}{d t}=A X(t)+B u(t)
$$

where $X$ is the state vector, $u$ is control vector, $A$ is the state matrix and $B$ is the input matrix. For the application in this paper, matrix $A$ has order 4 and matrix $B$ is $4 \times 2$, which will be defined later.

The LQR (Dorf and Bishop 2001) is an optimal control method that aims to find a control law $u(t)=-K X(t)$ that minimizes the square index $J^{*}$, given by Eq. 7: 


$$
J^{*}=\int_{0}^{\infty}\left(X^{T} Q X+u^{T} R u\right) d t
$$

where $Q$ is a positive, symmetric and semi defined real matrix (which represents the influence of states); and $R$ must be constant and positive (representing the influence of the control). In this application, matrices $Q$ and $R$ were considered identity matrices of order 4 and 2 , respectively.

Dorf and Bishop (2001) proposed an analytical solution to obtain the $K$ gain of the control law (Eq. 8):

$$
K=R^{-1} B^{T} P
$$

being $P$ the positive solution of Riccati equation (Eq. 9):

$$
A^{T} P+P A-P B R^{-1} B^{T} P+Q=0
$$

Then, the control law is given by Eq. 10:

$$
u(t)=-R^{-1} B^{T} P X(t)
$$

For a linear system such as that described by Eq. 6, both matrices A and B and the gain $K$ are constant, calculated only once at the beginning of the control project, and used for each instant of time throughout the application of the control.

In order to apply the LQR controller to the movement of the STS, an approximation of the state equations around the point of interest must be obtained. In the case, the point of interest is shown in Eq. 11:

$$
\left(\alpha, \beta, \frac{d \alpha}{d t}, \frac{d \beta}{d t}\right)=(0,0,0,0)
$$

As the sub satellite is stationary in relation to the main satellite, using the MacLaurin Series, it is possible to establish the Eqs. 12 and 13:

$$
\begin{gathered}
\frac{d^{2} \alpha}{d t^{2}}=F_{1}\left(\alpha, \beta, \frac{d \alpha}{d t}, \frac{d \beta}{d t}\right)=-2 \frac{M_{23}}{l}\left(\frac{d l}{d t}\right)\left(\frac{d w}{d t}\right)-3 \\
\frac{d^{2} \beta}{d t^{2}}=F_{2}\left(\alpha, \beta, \frac{d \alpha}{d t}, \frac{d \beta}{d t}\right)=-4
\end{gathered}
$$

The equations of motion, according to Denney and Greene (1991), linearized around the point of interest, constitute a nonlinear system since the matrix of coefficients $A$ is dependent on the control variable $\frac{d l}{l d t}$. Equations were linearized only around state variables, but resulting equations are nonlinear since the matrix of coefficients $A$ is dependent on the control. However, this approach allows the equations be written in form of Eq. 6; therefore, the LQR methodology can be applied. For this reason, the proposed control system recalculates the $K$ gain and the matrices $A$ and $B$ as a function of $\frac{d l}{l d t}$ for each instant of time, that is, they are not constant.

An important consideration is that the linearized equation for $\frac{d^{2} \beta}{d t^{2}}$ does not have a term independent of the state vector, so it is not possible to control it using only the LQR method. Therefore, to control the STS, it was decided to apply an external torque $\left(T_{q}\right)$ to the main satellite so that the angle outside the plane $(\beta)$ tends to zero. This torque is, therefore, the second control variable. This external torque can be generated by a gas thruster system on board the sub satellite. To perform system control, the ratio 
$\left(\frac{T_{q}}{j}\right)$ is added to the linearized equation of $\frac{d^{2} \beta}{d t^{2}}$ at each instant, where $J$ is the moment of inertia of the subsatellite in relation to the $X_{0}$ axis of the coordinate system originating from the main satellite (Eq. 14):

$$
J=\frac{m_{T} l^{2}}{3}+\frac{m_{2}\left(\operatorname{larg}{ }^{2}+\operatorname{comp}^{2}\right)}{12}
$$

The dimensions are in meters and described by Yong et al. (2009) as comp $=2$ and larg $=0.37037$.

Thus, in this case the control vector $(u(t))$ is given by Eq. 15:

$$
u(t)=\left[\begin{array}{c}
\frac{d l}{l d t} \\
T_{q}
\end{array}\right]
$$

Then, the controlled dynamic system is represented in the matrix form by Eq. 16:

$$
\left[\begin{array}{c}
\dot{\alpha} \\
\dot{\beta} \\
\ddot{\alpha} \\
\ddot{\beta}
\end{array}\right]=\left[\begin{array}{cccc}
0 & 0 & 1 & 0 \\
0 & 0 & 0 & 1 \\
-3\left(\frac{d w}{d t}\right)^{2} & 0 & -2 \frac{M_{23}}{l}\left(\frac{d l}{d t}\right) & 0 \\
0 & -4\left(\frac{d w}{d t}\right)^{2} & 0 & -2 \frac{M_{23}}{l}\left(\frac{d l}{d t}\right)
\end{array}\right]\left[\begin{array}{c}
\alpha \\
\beta \\
\dot{\alpha} \\
\dot{\beta}
\end{array}\right]+\left[\begin{array}{cc}
0 & 0 \\
0 & 0 \\
-2 M_{23}\left(\frac{d w}{d t}\right) & 0 \\
0 & \frac{1}{J}
\end{array}\right]\left[\begin{array}{c}
\frac{1}{l}\left(\frac{d l}{d t}\right) \\
T_{q}
\end{array}\right]
$$

\section{RESULTS}

The control system is implemented with the MATLAB software, with the following initial conditions (Eq.17):

$$
\left[\alpha ; \beta ; \frac{d \alpha}{d t} ; \frac{d \beta}{d t}\right]=\left[0^{\circ} ; 10^{\circ} ; 0,13 \% / s ; 0^{\circ} / s\right]
$$

Figures 3, 4 and 5 show the behavior of the applied torques $T_{q}$, of the control $\frac{d l}{l d t}$ and of the state variables, after applying the LQR method together with the torque, respectively. By the analysis of the values in graphs, it is possible to conclude that, after $200 \mathrm{~s}$, values of $\alpha$ and $\beta$ reached their final values, that is, they both came to zero. These results allow to conclude that the movement of the STS stabilizes after $200 \mathrm{~s}$, in the desired position (Eq. 18):

$$
X_{0}=\left(\alpha, \beta, \frac{d \alpha}{d t}, \frac{d \beta}{d t}\right)=(0,0,0,0) \text {, }
$$

restricting the movement of the subsatellite around the main satellite to the stretching of the tether in the orbital plane of the main satellite. 

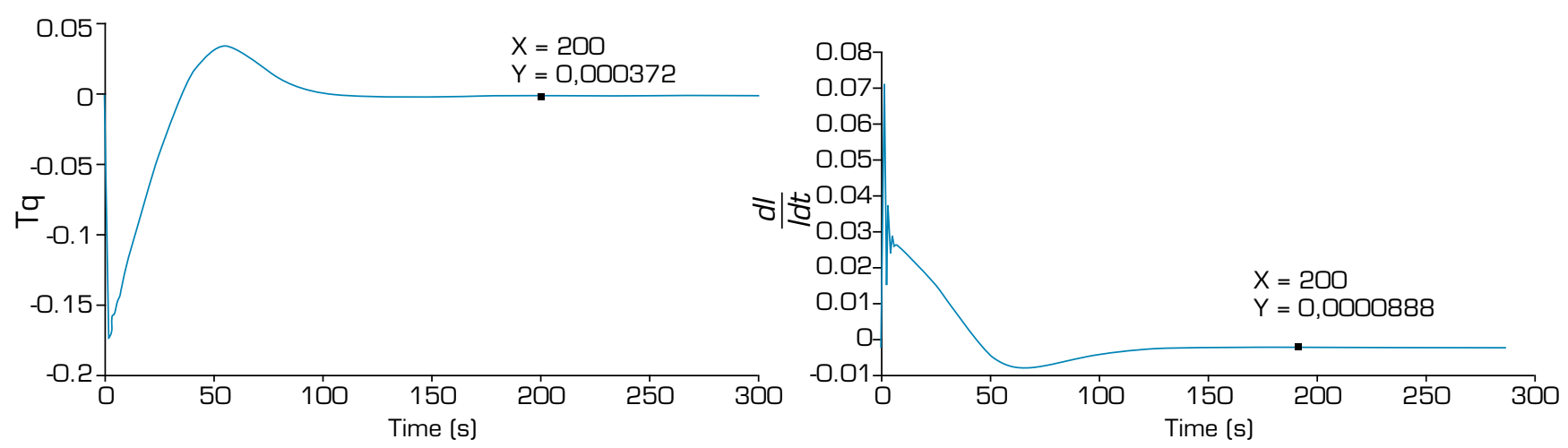

Figure 3. Temporal evolution torque $T_{q}$ and control $\frac{d l}{l d t}$ applied to STS, for $300 \mathrm{~s}$.
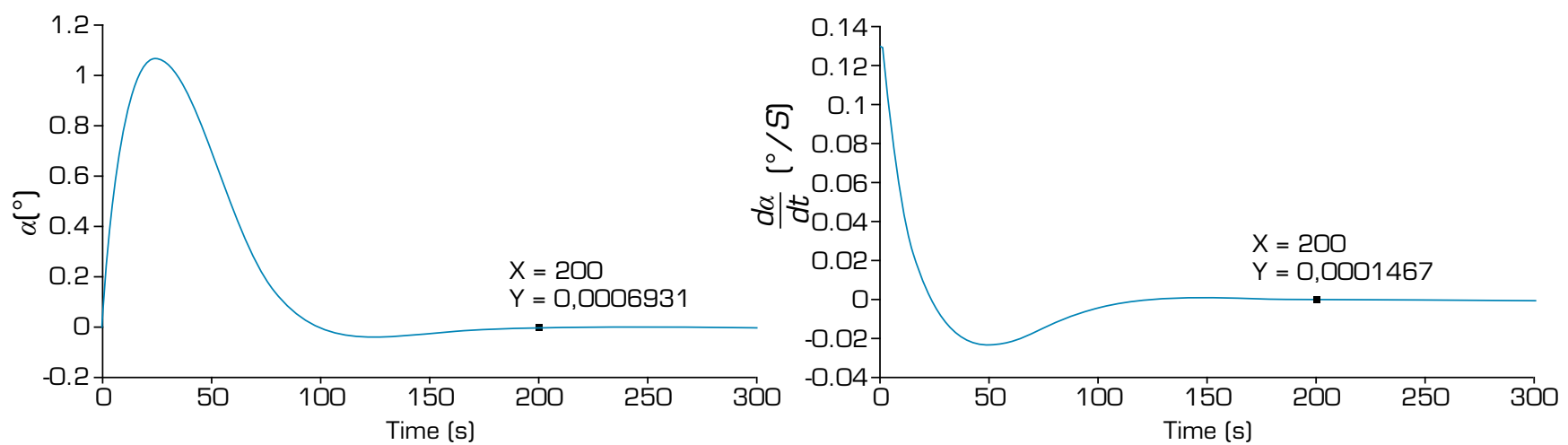

Figure 4. State variables $\alpha$ and $\frac{d \alpha}{d t}$ after the application the control LQR and torque for $300 \mathrm{~s}$.
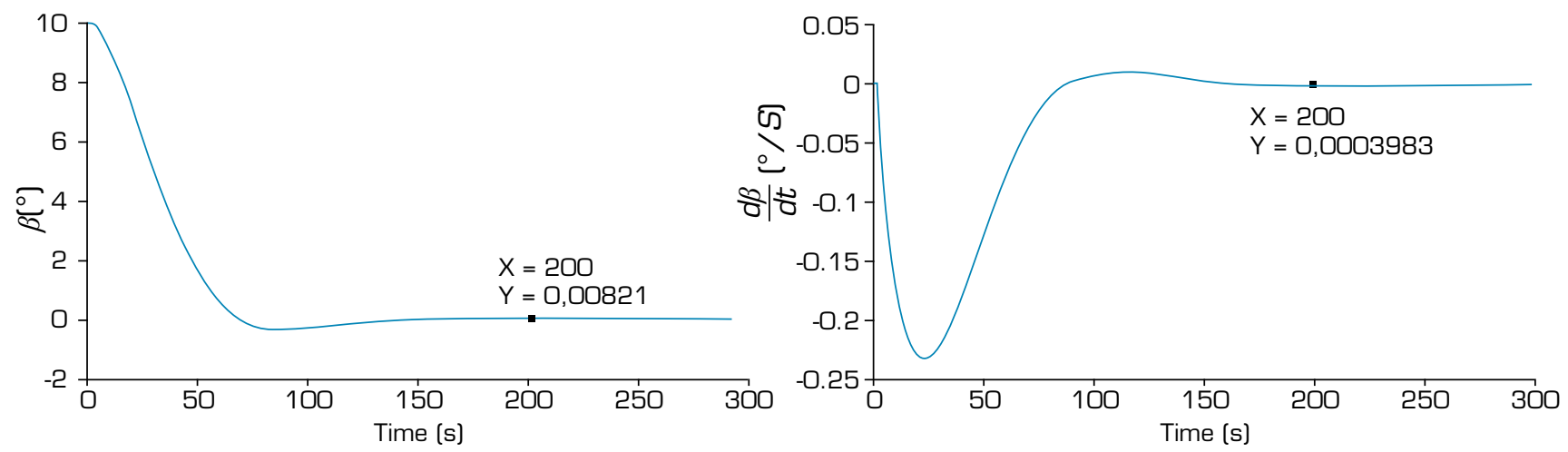

Figure 5. State variables $\beta$ and $\frac{d \beta}{d t}$ after the application the control LQR and torque for $300 \mathrm{~s}$.

Figure 6 illustrates the movement of the STS after the application of the LQR control, showing the initial and final position, as well as the sub satellite trajectory and the variation of the tether length. It is possible to detail the analysis of the movement, bringing the lateral and upper view, where the final angles in and outside the plane are explained, both null in the final instant, as shown in Figs. 7 and 8. 


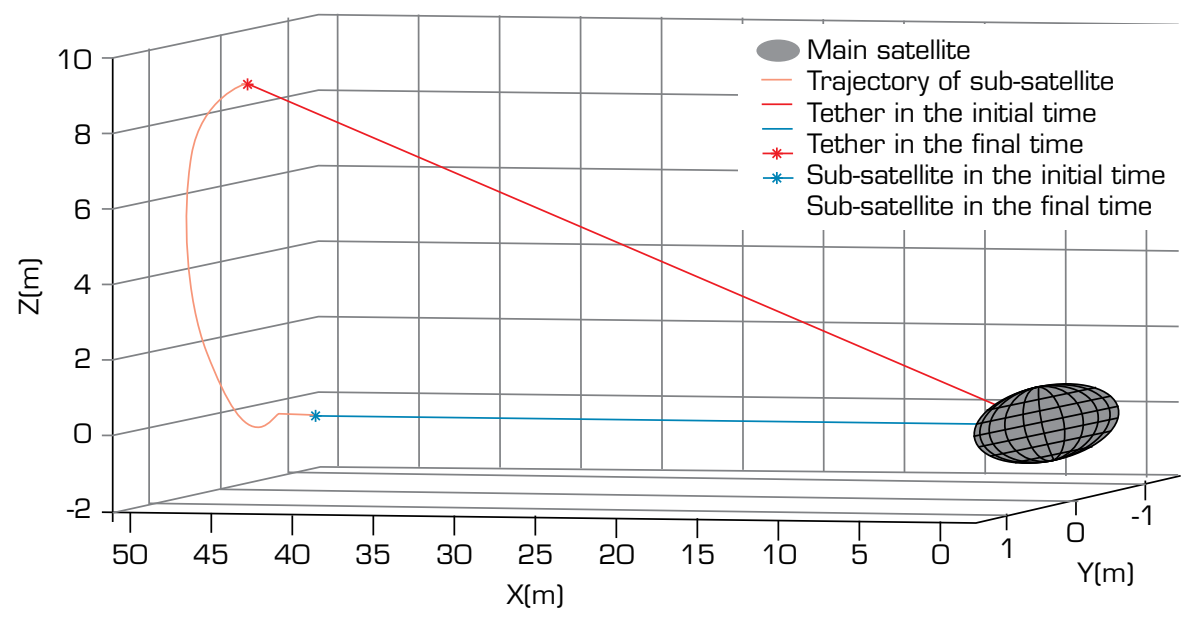

Figure 6. Three-dimensional representation of the movement of the controlled STS.
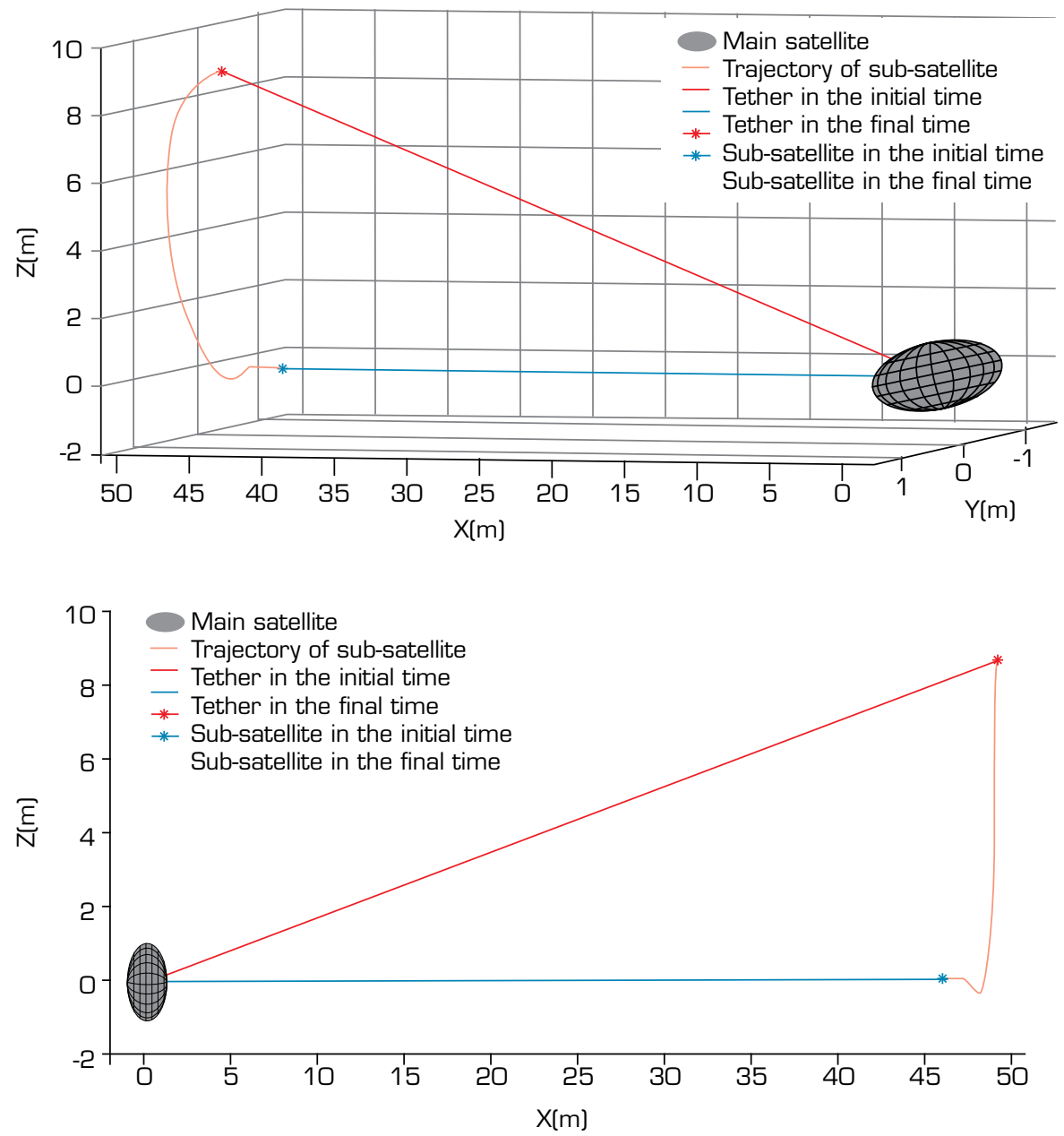

Figure 7. Side view of the movement of the controlled tether system, in yellow the tether at the final instant with angle $\beta=0^{\circ}$. 


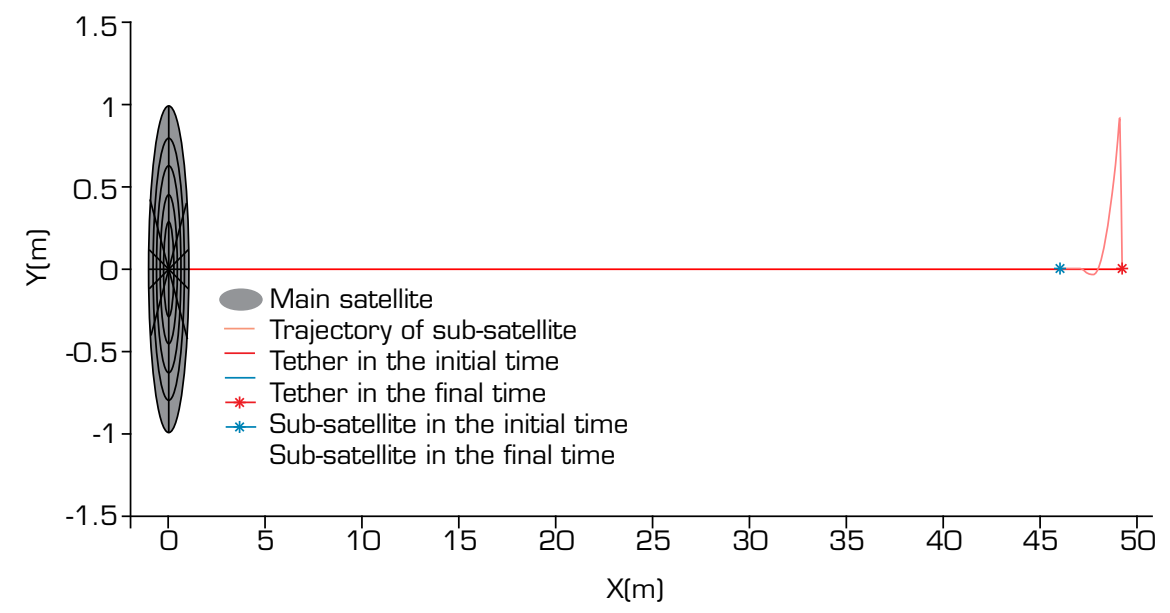

Figure 8. Upper view of the movement of the controlled tether system. It is possible to note the final position of the tether when $\alpha=0^{\circ}$.

Figure 9 illustrates the variation in the length of the tether for $300 \mathrm{~s}$ after the application of the LQR control, with the angles $\alpha=0^{\circ}$ and $\beta=0^{\circ}$, the tether is still retracting until reaching the desired $45 \mathrm{~km}$ in $1200 \mathrm{~s}$.

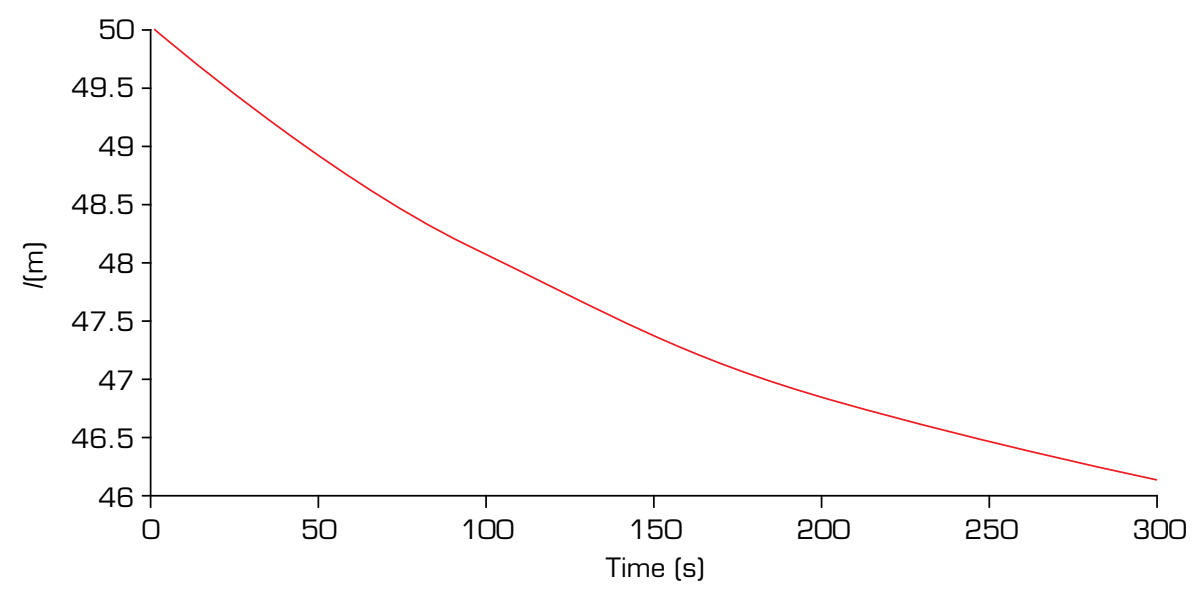

Figure 9. Temporal variation of tether length $l$ for 300 s.

\section{CONCLUSIONS}

By the analysis of the values it is possible to conclude that, after $200 \mathrm{~s}$, the results are within the tolerance values. The final results allow to conclude that the movement of the STS stabilizes after $200 \mathrm{~s}$, in the desired position $X_{0}=\left(\alpha, \beta, \frac{d \alpha}{d t}, \frac{d \beta}{d t}\right)=(0,0,0,0$,$) ,$ restricting the movement of the subsatellite around the main satellite to the stretching of the tether in the orbital plane of the main satellite. Figures 6 and 7 show the final position of the tether with an angle in $\left(\alpha=0^{\circ}\right)$ and outside the plane $\left(\beta=0^{\circ}\right)$.

This configuration is interesting because it allows the equipment and sensors of the subsatellite to remain pointed at the object of study of interest. For example, an STS orbiting Saturn in order to study it must keep its sensors always pointed towards the planet; in case it suffers a disturbance in its movement for whatever reason, it might be necessary to implement a control system similar to that proposed in this paper. 
Thus, the results show an effective control, with the motion of the subsatellite limited only to the stretch of the cable that links both satellites. However, it is necessary to introduce an auxiliary torque, since the linearized equation associated with the second variation of the angle out of the plan does not have a term independent of the state vector. This external torque can be generated by a gas thruster system on board the subsatellite.

The importance of discussing satellite systems connected by tethers is due to the vast range of their applications in several aerospace knowledge areas. The LQR method is used to implement a control system. This shows that a control system can be adapted to keep the movement in the plane. Also, others methods can be useful to construct the control. A similar control system could be help in others space missions.

\section{AUTHOR'S CONTRIBUTIONS}

Conceptualization: Zanardi MCand Baroni L; Supervision: Zanardi MCand Baroni L; Writing - Review \& Edition: Zanardi MC; Formal Analysis: Baroni L; Data Curation: Prado PR; Software: Prado PR; Investigation: Prado PR; Validation: Prado PR.

\section{DATA AVAILABILITY STATEMENT}

All the datasets were generated and analyzed during the current study.

\section{FUNDING}

Conselho Nacional de Desenvolvimento Científico e Tecnológico - Grant No: 421672/2016-1

http://dx.doi.org/10.13039/501100003593

\section{ACKNOWLEDGMENTS}

The authors are grateful to the Organization Committee of II Congresso Aeroespacial Brasileiro

\section{REFERENCES}

Cartmell MP, McKenzie DJ (2008) A review of space tether research. Prog Aerosp Sci 44(1):1-21. https://doi.org/10.1016/j. paerosci.2007.08.002

Chen Y, Huang R, Ren X, He L, He Y (2013) History of the Tether Concept and Tether Missions: A Review. Int Sch Res Notices 2013:502973. https://doi.org/10.1155/2013/502973

Cosmo ML, Lorenzini EC (1997) Tethers is Space Handbook. Cambridge: Smithsonian Astrophysical Observatory.

Dorf RC, Bishop RH (2001) Sistemas de Controle Moderno. Rio de Janeiro: LTC.

Ferreira AFS, Prado AFBA, Guerman A, Santos DPS, Burov A, Winter OC (2017a) Dynamics of a space tether in binary asteroids. Adv Astronaut Sci 162:3883-3902. 
Ferreira AFS, Prado AFBA, Guerman A, Santos DPS, Burov A, Winter OC (2017b) Using tethers to build a capture portal for the planets. Adv Astronaut Sci 162:3839-3858

Denney TS, Greene ME (1991) On state estimation for an orbiting single tether system. IEEE Trans Aerosp Electron Syst 27(4):689-695. https://doi.org/10.1109/7.85043

Lanoix, EL-M, Misra AK (2012) Near Earth asteroid missions using tether sling shot assist. J Spacecr Rockets 37(4):475-480. https://doi.org/10.2514/2.3588

Moia, GP, Santos DPS, Formiga JKS (2019) Tension force in nanosatellite tethers systems. J Phys Conf Ser 1365(2019):012010. https://doi.org/10.1088/1742-6596/1365/1/012010

Yong H, Bin L, WenFu X, Cheng L (2009) The research of real-time estimation method on tether parameters for tethered satellite. Paper presented 2009 International Conference on Information and Automation. Zhuhai, Macau, China. https://doi.org/10.1109/ ICINFA.2009.5204910

Zanardi MC, Livio B, Prado PR, Silva WR, Baroni L (2019) Dynamic propagation of space tether system motion. J Phys Conf Ser 1365(2019):012006. https://doi.org/10.1088/1742-6596/1365/1/012006 